\title{
Bronchoscopic administration of bovine natural surfactant in ARDS and septic shock: impact on biophysical and biochemical surfactant properties
}

\author{
A. Günther, R. Schmidt, J. Harodt, T. Schmehl, D. Walmrath, C. Ruppert, F. Grimminger, W. Seeger
}

\begin{abstract}
Bronchoscopic administration of bovine natural surfactant in ARDS and septic shock: impact on biophysical and biochemical surfactant properties. A. Günther, R. Schmidt, J. Harodt, T. Schmehl, D. Walmrath, C. Ruppert, F. Grimminger, W. Seeger. (C)ERS Journals Ltd 2002.

ABSTRACT: The purpose of the present study was to investigate the impact of bronchoscopic surfactant administration, on the biochemical and biophysical surfactant properties, in patients with severe and early acute respiratory distress syndrome (ARDS) and septic shock.

A total number of 27 ARDS patients received 300-500 $\mathrm{mg} \cdot \mathrm{kg} \cdot \mathrm{body} \cdot \mathrm{weight}^{-1}$ of a natural bovine surfactant extract (Alveofact $\AA$ ) via a flexible bronchoscope. Bronchoalveolar lavages were performed $3 \mathrm{~h}$ prior to, and $15-18 \mathrm{~h}$ and $72 \mathrm{~h}$ after surfactant administration. A comparison to healthy volunteers, undergoing an identical lavage procedure, was made (control, $\mathrm{n}=12$ ).

Severe biophysical and biochemical surfactant abnormalities were encountered throughout in the ARDS patients. These included a massive alveolar protein load, a reduced percentage of large surfactant aggregates (LA), a loss of palmitoylated phosphatidylcholine species and a significant reduction of surfactant apoprotein (SP)-A, SP-B and SP-C in the LA fraction. Both minimum ( $\gamma \mathrm{min})$ and adsorption ( $\gamma$ ads) surface tension values (pulsating bubble surfactometer) were dramatically increased. Surfactant treatment resulted in a marked increase in the lavagable phospholipid (PL) pool, but predominance of the alveolar surfactant-inhibitory protein load was still encountered. Far-reaching or even complete normalization of the PL profile, the LA fraction and its SP-B and SP-C (but not SP-A) content as well as the fatty acid composition of the phosphatidylcholine class was noted. Surface tension lowering properties ( $\gamma$ min and $\gamma$ ads) significantly improved, but were still not fully normalized.

Bronchoscopic administration of large quantities of natural bovine surfactant in severe acute respiratory distress syndrome causes far-reaching restoration of biochemical surfactant properties and significant improvement, however not full normalization, of biophysical surfactant function.
\end{abstract}

Eur Respir J 2002; 19: 797-804.

The acute respiratory distress syndrome (ARDS) is associated with pronounced biochemical and biophysical alterations of the pulmonary surfactant system, mostly assessed by analysis of the bronchoalveolar lavage fluid (BALF) [1-4]. These alterations include an increase in the total protein content of BALF, altered phospholipid (PL) and fatty acid profiles [5], reduced concentrations of the surfactant apoproteins (SP)-A and SP-B $[1,2]$ and a markedly low level of the biophysically most relevant large surfactant aggregate fraction (LA). As a consequence, a dramatic loss of surface activity was consistently noted in this disease [1-3]. Furthermore, degradation of essential surfactant compounds by inflammatory mediators (e.g. proteases, phospholipases) and inhibition of surfactant function by plasma proteins including fibrin(ogen) have been implicated in the impairment of surface activity. Loss of alveolar stability, atelectasis formation and severe deterioration of gas
Dept of Internal Medicine II, JustusLiebig-University, Giessen, Germany.

Correspondence: W. Seeger

Medizinische Klinik II

Zentrum für Innere Medizin

Justus-Liebig-University

Klinikstr. 36, D-35392 Giessen

Germany

Fax: 496419942359

E-mail: Werner.Seeger@innere.med.

uni-giessen.de

Keywords: Acute respiratory distress syndrome

sepsis

surface tension

surfactant phospholipids

Received: May 92001

Accepted after revision January 92002

The present study was supported by the Deutsche Forschungsgemeinschaft. exchange are considered to result largely from such surfactant abnormalities in ARDS. Indeed, manoeuvres to directly affect the alveolar surfactant system reproduced the gas exchange disturbances in ARDS, with models including hypochloric acid aspiration [6], experimental pneumonia [7], sepsis [8], bronchoalveolar detergent administration [9] and repetitive lavage procedures [10].

In the infant respiratory distress syndrome (IRDS), being primarily characterized by a deficiency of pulmonary surfactant, timely administration of exogenous surfactant was shown to markedly improve survival [11]. In addition, it was found that a prophylactic treatment was superior to delayed treatment, when ventilator associated inflammatory changes such as leukocyte and plasma protein influx into the alveolar compartment and increased endothelial and epithelial permeability are already evident. As in the animal models of ARDS, surfactant therapy under 
these conditions may be limited by the presence of inhibitory factors (proteases, phospholipases, radicals, proteins), demanding an increase in the surfactant dosage to overcome the inhibitory capacities in the alveolar space.

Despite such evidence for a role of surfactant abnormalities in ARDS and the unequivocal clinical efficacy of surfactant replacement in IRDS, only few attempts were hitherto undertaken to improve gas exchange in ARDS patients by exogenous surfactant administration [12-14]. Data concerning the impact of such surfactant-replacement therapy on the biochemical and biophysical surfactant properties are scarce and limited to rather late time periods after performance of the replacement therapy, with only minor changes being observed [12]. Preliminary data from the authors' group [14] in a small number of ARDS patients (10) suggested rapid changes in surfactant function and composition after bronchoscopic administration of a calf-lung surfactant extract. The results of a multicentre noncontrolled study, addressing the feasibility and safety of a bronchoscopic administration of $300-500 \mathrm{mg} \cdot \mathrm{kg}^{-1}$ body weight (bw) of this natural bovine surfactant (Alveofact; Thomae $\mathrm{GmbH}$, Biberach, Germany), in 27 patients suffering from severe and early ARDS and septic shock are presented here. Due to the limited number of patients, the heterogeneity of septic ARDS, and the difficulty in obtaining BALF without therapeutic intervention no placebo-treated control group was incorporated. With the clinical and gas exchange responses being presented in the accompanying paper [15], in the present study the authors found that bronchoscopic surfactant treatment provoked a marked increase in the lavagable PL content and a far-reaching normalization of the PL-, fatty acid- and hydrophobic apoproteinprofiles and the percentage of large surfactant aggregates, which was linked to a significant improvement in the surface tension lowering properties.

\section{Methods}

\section{Patient population}

A total of 27 patients with severe ARDS according to the Consensus Criteria [16] were entered into the study between 1994-1996. Patients were considered for entry into the study if they: were aged 18-70 yrs; developed acute respiratory failure with typical bilateral infiltrates on the chest radiograph after a typical predisposing event (all patients included fulfilled the criteria septic shock); had a Murray score 2.5 [16], and had been ventilated $\leqslant 96 \mathrm{~h}$ since ARDS diagnosis (early ARDS). All criteria had to be fulfilled over a baseline period of $12 \mathrm{~h}$ preceding entry into the study. The following exclusion criteria were checked in all patients: pregnancy; acute myocardial infarction; left heart failure (pulmonary capillary wedge pressure $>18 \mathrm{mmHg}$ as assessed by a pulmonary-artery catheter); lung contusion; pre-existing pulmonary diseases (fibrosis, chronic obstructive lung disease); malignant underlying disease; immunosuppressive drugs and leukopenia (white blood cells
$<1,000 \mu \mathrm{L}^{-1}$ ); severe traumatic or hypoxic brain injury; additional investigational drugs. Details on ventilator settings are given in detail in [15]. In brief, the mean \pm SEM tidal volume was $11.0 \pm 0.5 \mathrm{~mL} \cdot \mathrm{kg}^{-1}$ bw and positive end-expiratory pressure was $16.3 \pm$ $2.8 \mathrm{cmH}_{2} \mathrm{O}$. The institutional ethics committee at each centre approved the study protocol. Each patient, or their legal guardian, gave informed consent before study entry. For comparison, 12 healthy volunteers without any clinical signs or history of heart or lung disease underwent flexible bronchoscopy and bronchoalveolar lavage, but not ventilation and sham exposure.

\section{Surfactant material and bronchoscopic administration}

The study was performed with Alveofact, a bovine natural surfactant. It is a chloroform/methanol extract of calf lungs containing PLs, neutral lipids and the hydrophobic SP-B and SP-C (see later); it does not contain substantial quantities of the hydrophilic apoproteins SP-A and SP-D. Time was set at zero after baseline and $300 \mathrm{mg} \cdot \mathrm{kg}^{-1}$ bw of Alveofact was bronchoscopically delivered, in divided doses, to each segment of the right and left lung (in total $22.7 \pm 3.4 \mathrm{~g}$ in $\sim 378 \mathrm{~mL}$ saline). Starting from the lower segments, the bronchoscope was wedged in each single segment for injection of the aliquot, followed by $1-2 \mathrm{~min}$ maintenance of the wedge position to avoid a major reflux of the surfactant material into the central airways. After each 2-3 segments in one lung, the procedure was continued in the other lung. The overall treatment procedure lasted $45 \pm 11 \mathrm{~min}$. During the period of surfactant administration, the supine position of the patient and the ventilator settings were not changed. In those patients, in whom the bronchoscopic surfactant administration caused an improvement of the oxygen tension in arterial blood/inspiratory oxygen fraction $\left(\mathrm{Pa}, \mathrm{O}_{2} / \mathrm{FI}, \mathrm{O}_{2}\right)$ of at least $25 \%$, which was however, lost again over the next 18-24 h, retreatment with an additional surfactant dose of $200 \mathrm{mg} \cdot \mathrm{kg}^{-1}$ bw was allowed $18-24 \mathrm{~h}$ after the first surfactant administration. This was performed in seven out of the 27 patients (total second dose of $14.0 \pm 0.44 \mathrm{~g}$ in $\sim 230 \mathrm{~mL}$ saline).

\section{Bronchoalveolar lavage}

Bronchoalveolar lavages were performed $3 \mathrm{~h}$ prior, as well as, $15-18 \mathrm{~h}$ and $72 \mathrm{~h}$ after surfactant administration. Flexible fibreoptic bronchoscopy was undertaken in a standardized manner as previously described [1]. One segment of the lingula or the right middle lobe was lavaged with a total volume of $200 \mathrm{~mL}$ of sterile saline in 10 aliquots with a fluid recovery ranging between $50-70 \%$. The fractions of the BALF were pooled, filtered through sterile gauze, and centrifuged at $200 \times g\left(10 \mathrm{~min}, 4^{\circ} \mathrm{C}\right)$ to remove cells and membranous debris. The supernatant was immediately divided into aliquots, frozen in liquid nitrogen and stored at $-80^{\circ} \mathrm{C}$ until further processing. 


\section{Phospholipid analysis}

Lipids were extracted from BALF with chloroform/ methanol [17], and the PL content was determined by spectrophotometric measurement of phosphorus according to the method of Rouser et al. [18]. Individual PLs were separated by high-performance thin-layer chromatography (TLC) as previously described in detail [1] using silica 60 plates (Merck, Darmstadt, Germany) and chloroform:methanol: acetic acid:water (50:37.5:3.5:2; per cent volume in volume $(\mathrm{v} / \mathrm{v}))$ as the developing solvent and a molybdenum blue reagent for staining [19]. Quantification was carried out by means of densitometric scanning at $700 \mathrm{~nm}$ using a TLC-scanner II (Camag, Berlin, Germany). Fatty acid composition of phosphatidylcholine (PC) was determined as described recently [5]. For this purpose, PC was separated as described earlier, visualized with primuline [20], eluted off the silica matrix after addition of $10 \mu \mathrm{g}$ pentadecanoic acid as an internal standard and underwent acid-catalyzed transmethylation with $2 \mathrm{~N}$ $\mathrm{HCl}$ for $12 \mathrm{~h}$ at $100^{\circ} \mathrm{C}$. The resulting fatty acid methyl esters (FAME) were analysed by gas chromatography (Carlo Erba Fractovap 2150, Mainz, Germany). The individual FAME were detected by means of a flame ionisation detector and identified by comparison with the retention times of commercially available standards (Sigma, Deisenhofen, Germany).

\section{Protein analysis}

Total BALF protein was measured using a commercial assay (BCA assay, Pierce, Rockford, IL, USA). SP-A and SP-B were analysed in the isolated large surfactant aggregates using enzyme-linked immunosorbent assay (ELISA) techniques as previously described $[1,21]$. The SP-C was determined using a recently developed ELISA technique [22].

\section{Isolation and quantification of large surfactant aggregates}

Aliquots of BALF were centrifuged at $48,000 \times g$ (for $1 \mathrm{~h}$, at $4^{\circ} \mathrm{C}$ ), thereby separating the large from the small surfactant aggregates $[2,23]$. The pellet representing the LA was resuspended in a small volume of $0.9 \%$ saline $/ 3 \mathrm{mM} \mathrm{CaCl} 2$ for determination of the PL content. The relative content of LA was expressed as a per cent of the total PL.

\section{Surface tension measurements}

The pellets were adjusted to a concentration of $2 \mathrm{mg} \cdot \mathrm{mL}^{-1} \mathrm{PL}$, vortexed for $1 \mathrm{~min}$, and subjected to surface tension measurements, which were performed by means of a pulsating bubble surfactometer (Electronetics, NY, USA) as previously described $[24,25]$. The surface tension values after $5 \mathrm{~min}$ of film oscillation at the minimum bubble radius $(\gamma \mathrm{min})$ and after 11-s film adsorption ( $\gamma$ ads) are given. The inhibitory effect of the $48,000 \times g$ supernatant proteins on the surface activity was investigated by reconstitution of LA (dissolved at a final PL concentration of $2 \mathrm{mg} \cdot \mathrm{mL}^{-1}$ ) with the BALF proteins at the same ratio as observed in the original lavage fluid, as previously described in detail [1].

\section{Statistics}

All values are presented as mean \pm SEM. Statistical analysis was performed by testing firstly, the normal distribution (Kolmogorov-Smirnov-test), followed by a pairwise comparison of the baseline and postsurfactant data either with a paired t-test (in the case of normal distribution) or with Wilcoxon's matchedpairs signed-ranks test. Values significantly different from presurfactant values were indicated.

\section{Results}

In ARDS, the BALF protein levels were increased 25-fold as compared to the controls, whereas the total PL concentrations were largely unchanged (table 1). Thus, the PL-protein ratio, reflecting the burden of putatively surfactant inhibitory proteins within the alveolar compartment, was lowered by more than one order of magnitude.

In addition, marked changes of the biophysical and biochemical surfactant properties were encountered in the ARDS patients prior to surfactant application. The relative amount of large surfactant aggregates was markedly decreased to $\sim 36 \%$ of total PLs, as

Table 1. - Protein and phospholipid content of bronchoalveolar lavage fluid ${ }^{\#}$

\begin{tabular}{lcccc}
\hline & \multicolumn{3}{c}{ ARDS patients } & Control \\
\cline { 2 - 4 } & $3 \mathrm{~h}$ pre & $15-18 \mathrm{~h}$ post & \multirow{2}{*}{$\mathrm{h}$ post } \\
\hline Subjects $\mathrm{n}$ & 27 & 25 & 22 & 12 \\
Protein $\mathrm{mg} \cdot \mathrm{mL}^{-1}$ & $1.70 \pm 0.45$ & $1.12 \pm 0.35$ & $0.92 \pm 0.24^{* *}$ & $0.07 \pm 0.01$ \\
PL $\mu \mathrm{g} \cdot \mathrm{mL}^{-1}$ & $29.9 \pm 6.1$ & $129.2 \pm 19.9^{* * *}$ & $80.6 \pm 20.3^{* * *}$ & $26.4 \pm 2.32$ \\
PPQ & $0.041 \pm 0.013$ & $0.271 \pm 0.047^{* * *}$ & $0.131 \pm 0.043^{* * *}$ & $0.470 \pm 0.062$ \\
\hline
\end{tabular}

Data are presented as mean \pm SEM. * : Original lavage fluids were analysed in acute respiratory distress syndrome (ARDS) patients (pre- and postsurfactant administration) and in healthy controls. Data are given for protein and phospholipid (PL) content; additionally, the PL/protein quotient (PPQ) was calculated. ${ }^{* *}: \mathrm{p}<0.01 ; * *: \mathrm{p}<0.001$ as compared to baseline values ( $3 \mathrm{~h}$ pre). 


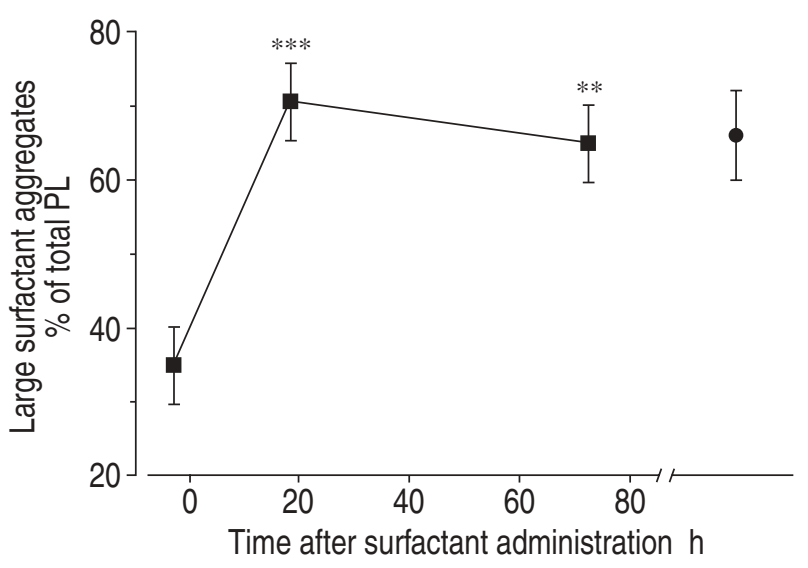

Fig. 1.-Relative content of large surfactant aggregates, given as a per cent of all phospholipids (PL). Data are presented as the meanSEM values of the patients $(\boldsymbol{O})$ and healthy controls $(\boldsymbol{\square}){ }^{* *}$ : $\mathrm{p}<0.01, * * *: \mathrm{p}<0.001$ as compared to baseline values $(3 \mathrm{~h}$ preadministration).

compared to $\sim 60-75 \%$ in the healthy controls (fig. 1). The PL profile was characterized by a decrease in the relative concentration of $\mathrm{PC}$ and phosphatidylglycerol (PG) and a concomitant increase in the relative amount of phosphatidylinositol, phosphatidylethanolamine, phosphatidylserine and sphingomyelin (table 2). In addition, the fatty acid profile of PC, representing the most abundant single surfactant component, was significantly altered, with a decrease in the palmitoylated species from $\sim 72 \%$ of total PC in controls to $\sim 60 \%$ in the ARDS patients (table 2 ). In addition, there was an increase in the relative amount of unsaturated PC fatty acids (oleic acid, linoleic acid). Concerning the SP, a marked loss of SP-A, SP-B and SP-C within the LA fraction was observed in all ARDS patients (figs. 2a-c). In detail, these apoproteins were reduced to $\sim 30 \%$ (SP-A, fig. 2a), $\sim 20 \%$ (SP-B, fig. 2 b) and $\sim 45 \%$ (SP-C, fig. 2c) as compared to the control values. In parallel, the surface activity of the isolated LA fraction, although being tested at a preset PL concentration of $2 \mathrm{mg} \cdot \mathrm{mL}^{-1}$, was significantly impaired (figs. 3a and b). The surface tension after 12-s rads, (fig. 3b) was found to be $\sim 22 \mathrm{mN} \cdot \mathrm{m}^{-1}$ in the controls, but was up to $\sim 44 \mathrm{mN} \cdot \mathrm{m}^{-1}$ in the ARDS patients. Accordingly, the $\gamma \mathrm{min}$ (fig. 3a) was found to be near $0 \mathrm{mN} \cdot \mathrm{m}^{-1}$ in the controls, but was significantly elevated to $\sim 23 \mathrm{mN} \cdot \mathrm{m}^{-1}$ in the patients. Furthermore, when recombining the LA fractions with the supernatant proteins isolated from the same BALF, at the ratio detected in the original lavage fluids, a further deterioration of surfactant function was noted (figs. 4a and $\mathrm{b}$ ), with $\gamma$ min values increasing to $\sim 31 \mathrm{mN} \cdot \mathrm{m}^{-1}$ (again near zero in controls; fig. 4a).

In response to surfactant treatment, the total lavagable PL pool increased greater than four-fold, as assessed after 15-18 h, and was still elevated nearly three-fold after $72 \mathrm{~h}$ (table 1). As a result, the PL-protein-ratio, although not being fully normalized, was increased by several fold. The large surfactant aggregates reached control values both $15-18 \mathrm{~h}$ and $72 \mathrm{~h}$ after surfactant administration (fig. 1). Furthermore, the PL profile was virtually normalized by the endobronchial surfactant administration, with details of this profile in the postsurfactant lavages being very similar to the Alveofact profile (table 2). In contrast, the fatty acid profile of PC, though obviously responding to the surfactant administration and approaching the Alveofact profile, was not fully normalized in the postsurfactant lavages. As could be assumed on the basis of the apoprotein composition of Alveofact, there was a significant increase in SP-B and SP-C in the LA fraction in response to surfactant treatment, with values reaching the control range

Table 2. - Phospholipid composition of bronchoalveolar lavage fluid (BALF) and fatty acid profiles of phosphatidylcholine ${ }^{\#}$

\begin{tabular}{lccrrr}
\hline & \multicolumn{3}{c}{ ARDS patients } & Control & Alveofact巴 \\
\cline { 2 - 4 } & $3 \mathrm{~h}$ pre & $15-18 \mathrm{~h}$ post & $72 \mathrm{~h}$ post & \\
\hline Subjects n & 27 & 25 & 22 & 12 & 3 \\
PC \% & $72.3 \pm 2.1$ & $85.7 \pm 0.9^{* * *}$ & $83.5 \pm 1.0^{* * *}$ & $83.1 \pm 0.9$ & $82.1 \pm 0.9$ \\
PG \% & $3.2 \pm 0.6$ & $8.1 \pm 0.7^{* * *}$ & $6.9 \pm 0.9^{* * *}$ & $8.6 \pm 0.6$ & $9.0 \pm 0.8$ \\
PI \% & $7.6 \pm 1.0$ & $1.7 \pm 0.2^{* * *}$ & $2.7 \pm 0.3^{* * *}$ & $3.2 \pm 0.2$ & $0.5 \pm 0.2$ \\
SPH \% & $9.4 \pm 1.2$ & $1.9 \pm 0.2^{* * *}$ & $3.2 \pm 0.3^{* * *}$ & $0.8 \pm 0.2$ & $1.4 \pm 0.2$ \\
PE \% & $5.1 \pm 0.8$ & $2.0 \pm 0.3^{* * *}$ & $2.4 \pm 0.3^{* * *}$ & $1.7 \pm 0.2$ & $3.0 \pm 0.1$ \\
PS \% & $1.8 \pm 0.6$ & $0.5 \pm 0.2^{* * *}$ & $1.2 \pm 0.3$ & $1.2 \pm 0.3$ & 0.0 \\
$14: 0$ PC BALF \% & $1.56 \pm 0.73$ & $3.22 \pm 0.33$ & $3.78 \pm 0.28^{*}$ & $3.94 \pm 0.63$ & $3.65 \pm 0.08$ \\
$16: 0$ PC BALF \% & $59.92 \pm 5.77$ & $65.98 \pm 1.01$ & $67.55 \pm 2.04$ & $72.31 \pm 1.35$ & $73.45 \pm 0.69$ \\
$18: 0$ PC BALF \% & $5.34 \pm 3.28$ & $4.35 \pm 0.45$ & $4.24 \pm 0.53$ & $6.74 \pm 1.02$ & $4.34 \pm 0.02$ \\
$18: 1$ PC BALF \% & $19.39 \pm 2.11$ & $20.59 \pm 0.91^{*}$ & $16.71 \pm 1.36$ & $9.66 \pm 0.43$ & $15.47 \pm 0.50$ \\
$18: 2$ PC BALF \% & $9.34 \pm 2.29$ & $3.01 \pm 0.48$ & $3.28 \pm 0.49^{* *}$ & $3.95 \pm 0.29$ & $1.42 \pm 0.07$ \\
\hline
\end{tabular}

Data are presented as mean \pm SEM. PC: phosphatidylcholine; PG: phosphatidylglycerol; PI: phosphatidylinositol; SPH: sphingomyelin; PE: phosphatidylethanolamine; PS: phosphatidylserine. \#: Original lavage fluids were analysed in acute respiratory distress syndrome (ARDS) patients (pre- and postsurfactant administration) and in healthy controls. In addition, the bovine surfactant preparation (Alveofact $(\mathrm{B})$ was studied. Depicted is the relative content of each phospholipid class in per cent of all phospholipids. 14:0: myristic acid; 16:0: palmitic acid; 18:0: stearic acid; 18:1: oleic acid; 18:2: linoleic acid. Lyso-phosphatidylcholine and cardiolipin were found as trace amounts. The relative content of each fatty acid is given in per cent of all fatty acids analysed in PC isolated from original lavage fluid (BALF). Fatty acids with a relative distribution $<0.5 \%$ are not given. $*: \mathrm{p}<0.05 ; * *: \mathrm{p}<0.01 ; * * *: \mathrm{p}<0.001$ as compared to baseline values $(3 \mathrm{~h}$ pre). 

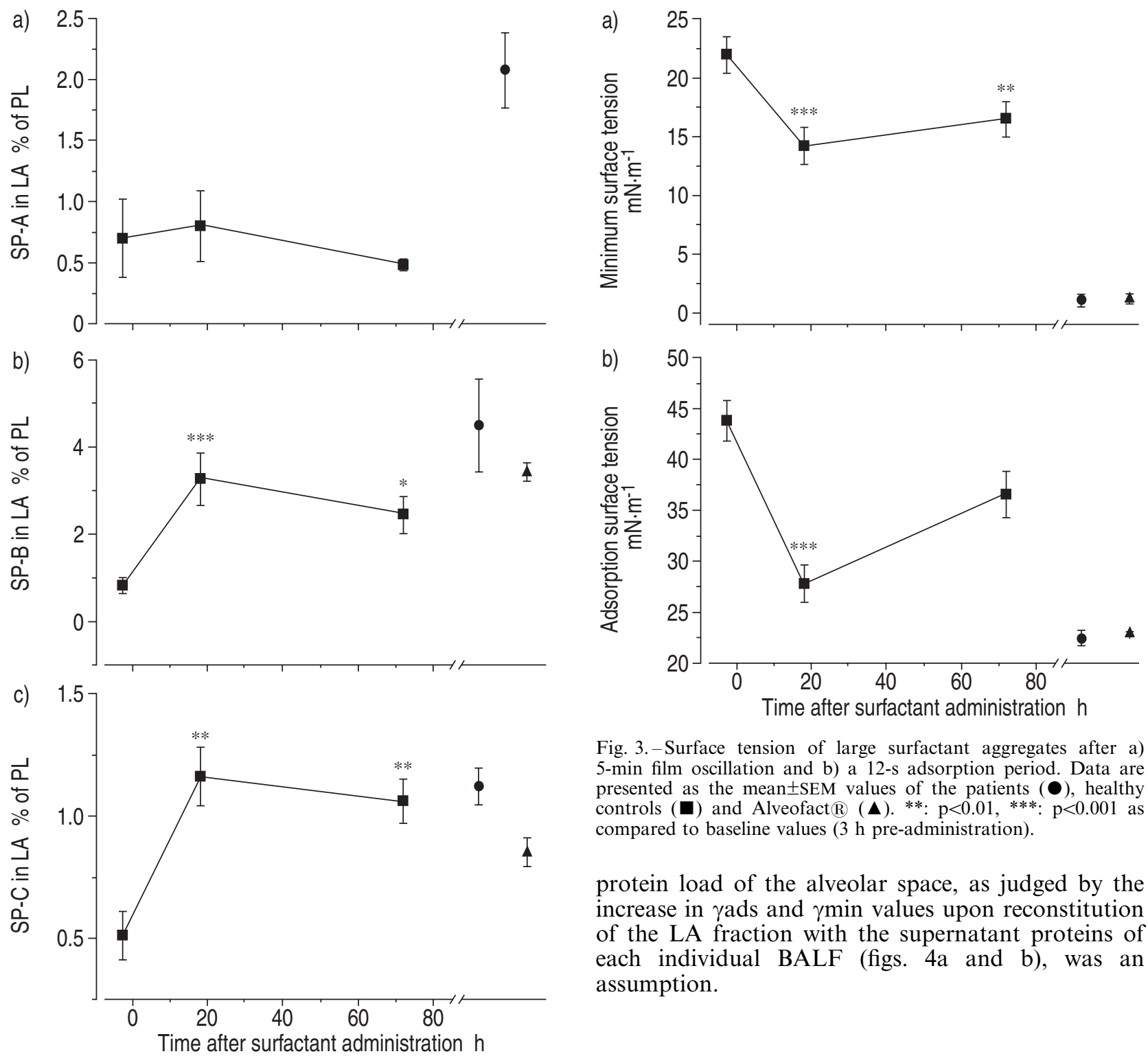

Fig. 3.-Surface tension of large surfactant aggregates after a) 5-min film oscillation and b) a 12-s adsorption period. Data are presented as the mean \pm SEM values of the patients $(0)$, healthy controls ( $\boldsymbol{\square})$ and Alveofact $\mathbb{R}(\boldsymbol{\Delta}){ }^{* *}: \mathrm{p}<0.01,{ }^{* * *}: \mathrm{p}<0.001$ as compared to baseline values ( $3 \mathrm{~h}$ pre-administration).

protein load of the alveolar space, as judged by the increase in $\gamma$ ads and $\gamma$ min values upon reconstitution of the LA fraction with the supernatant proteins of each individual BALF (figs. $4 \mathrm{a}$ and b), was an assumption.

Fig. 2.-Relative content of a) surfactant apoprotein (SP)-A b) SP-B and c) SP-C in large surfactant aggregates, given as a per cent of phospholipids (PL). Data are presented as the meanSEM values of the patients $(\boldsymbol{O})$, healthy controls $(\boldsymbol{\square})$ and Alveofact $\mathbb{R}$ $(\boldsymbol{\Delta}) .^{*}: \mathrm{p}<0.05 ; * *: \mathrm{p}<0.01, * * *: \mathrm{p}<0.001$ as compared to baseline values ( $3 \mathrm{~h}$ pre-administration).

(figs. $2 \mathrm{~b}$ and c). In contrast, the relative concentration of SP-A was found to be slightly decreased in the postsurfactant LA fraction. The absolute SP-A levels in the original BALF were unchanged (data not shown in detail).

The post-treatment biophysical activity of the LA fraction from the patients was markedly improved, with a decrease of the rads values to $\sim 27 \mathrm{mN} \cdot \mathrm{m}^{-1}$ $\left(15-18 \mathrm{~h}\right.$ post) and $\sim 36 \mathrm{mN} \cdot \mathrm{m}^{-1}$ (72 $\mathrm{h}$ post, fig. $\left.3 \mathrm{~b}\right)$. Similarly, the $\gamma \mathrm{min}$ values were decreased to $\sim 14 \mathrm{mN} \cdot \mathrm{m}^{-1}$ (18 h post) and $\sim 17 \mathrm{mN} \cdot \mathrm{m}^{-1}(72 \mathrm{~h}$ post, fig. 3a), suggesting significant improvement but not complete normalization of surface activity. In addition, inhibition of surfactant function by the

\section{Discussion}

In the present study, the biochemical composition and the surface tension lowering properties of surfactant isolates, from patients with severe septic and early ARDS, pre- and postbronchoscopic surfactant administration were investigated. As compared to healthy volunteers, most severe abnormalities of surfactant composition and function were encountered in these seriously ill patients. Both the rads and the $\gamma$ min values were drastically increased, although measurements were undertaken at a standardized PL concentration. All surfactant constituents hitherto described as essential in terms of surface activity were found to be substantially reduced. 1) There was a decrease in the fractions of PC and PG within the total PL pool, as previously reported for ARDS lungs [1-3]. Concomitantly, the percentages of the other PL classes were increased, in particular those of sphingomyelin, phosphatidylinositol and phosphatidylethanolamine. 2) Within the PC class, the percentage of 

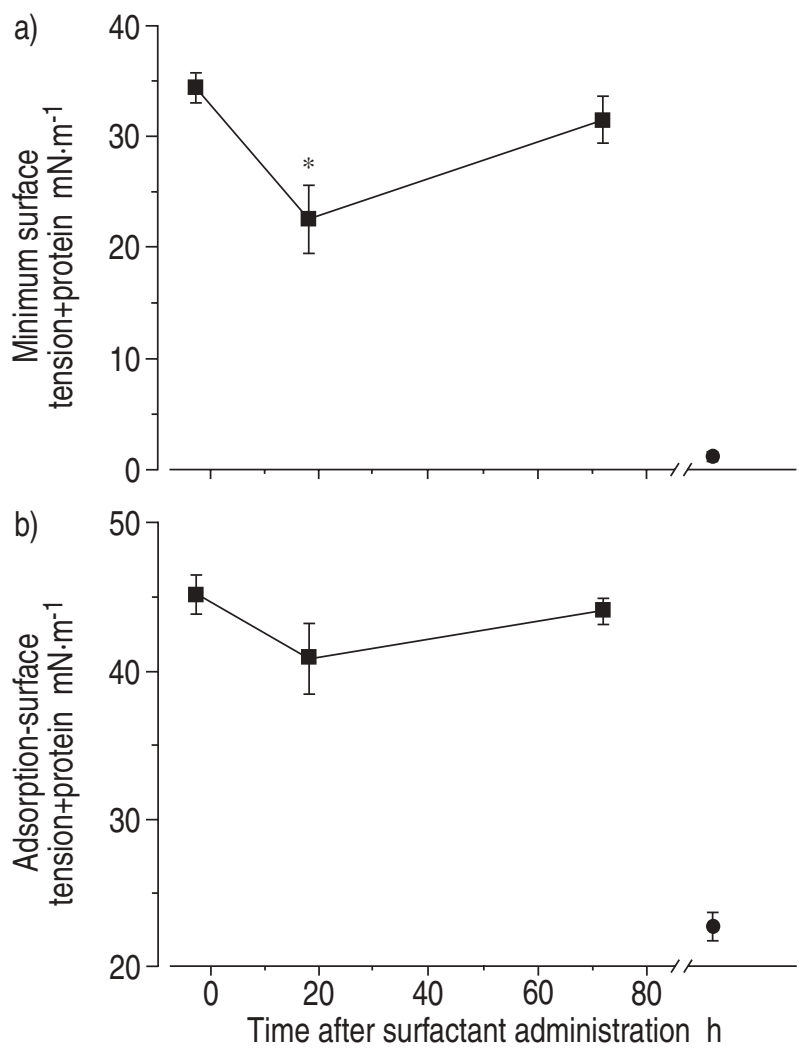

Fig. 4.-Inhibition of surface activity by supernatant bronchoalveolar lavage fluid proteins. The large surfactant aggregates were reconstituted with the corresponding amount of $48,000 \times g$ supernatant proteins. The surface tension after a) a 5-min film oscillation and b) a 12-s adsorption period. Data are presented as the

mean \pm SEM values of the patients $(\mathbf{O})$ and healthy controls $(\mathbf{\square})$.
$*: \mathrm{p}<0.05$ as compared to baseline values ( $3 \mathrm{~h}$ pre-administration).

palmitic acid was lowered. This finding supports the recent observation by the authors' group that a decrease in the palmitic acid content of PC was consistently noted in ARDS and severe pneumonia [5]. Moreover, preliminary analysis of the molecular species suggested that the percentage of dipalmitoylphosphatidylcholine (DPPC) is even more prominently reduced than the overall palmitic acid content in the PC pool [26]. This is a notable observation in view of the specific role of DPPC in establishing the highly condensed surfactant film during compression, a prerequisite for the very low minimal surface tension values. 3) In line with previous investigations $[1,27,28]$, a marked reduction of the large surfactantaggregate pool, the biophysically active fraction of the surface-active material in the alveolar compartment, was noted. The currently observed range of LA percentages, with a mean of $36 \%$ instead of $\sim 60-75 \%$ in controls, is indicative of the severity of ARDS the patients in the present study suffered from. 4) Within the LA fraction, the percentages of all apoproteins implicated in surface-tension regulation were markedly reduced. This was previously described for SP-A [2] and SP-B [2, 28], with the currently observed decline in SP-B to $<20 \%$ being most impressive. In addition, due to the establishment of a reliable ELISA technique for quantification of SP-C, this is the first study to report that the content of this hydrophobic apoprotein is also markedly reduced in the LA fraction of ARDS patients.

In addition to these changes in biochemical surfactant composition, an inhibitory effect of the soluble-lavage proteins was directly demonstrated by reconstituting the isolated LA fraction with the supernatant proteins originating from the same BALF. Altogether, these various changes in the surfactant system of the ARDS patients, although investigated ex vivo, indicate a marked loss of surfactant function under in vivo conditons also, which is in line with the severe gas exchange abnormalities that were observed in the patients, with predominance of shunt flow.

The bronchoscopic administration of $300-500 \mathrm{mg} \cdot \mathrm{kg}^{-1}$ bw of Alveofact $\mathbb{R}$, an organic solvent extract with excellent surface activity when tested in vitro [29] or in animal studies [30], resulted in a rapid and highly significant increase in $\mathrm{Pa}, \mathrm{O}_{2} / \mathrm{Fi}_{1} \mathrm{O}_{2}$ values from $\sim 110 \mathrm{mmHg}$ to $\sim 210 \mathrm{mmHg}$ [15]. The present study demonstrated that this is paralleled by an impressive restoration of surfactant properties, with respect to both the biochemical and the biophysical variables. Similar to the gas exchange response, the improvement of surfactant properties was even more prominent when re-assessed after $15-18 \mathrm{~h}$ than after $72 \mathrm{~h}$. In particular, the PL profile was virtually normalized, and this was also true for the percentage of LA and for the SP-C content in the LA fraction. The SP-B content within the LA pool was markedly increased, but did not fully reach control values. This was similarly true for the fatty acid composition of the PC class, in particular with respect to palmitic acid. The percentage of this fatty acid in the post-treatment lungs ranged between that of the pretreatment lavages and that of control lavages. Despite the fact that a rapid improvement of surfactant properties was achieved by the surfactant replacement regimen, several aspects deserve particular consideration.

Firstly, there was a greater than four-fold increase in the lavagable PL pool in response to surfactant administration. However, the amount of administered surfactant material $\left(300-500 \mathrm{mg} \cdot \mathrm{kg}^{-1} \mathrm{bw}\right)$ surpassed the endogenous surfactant pool $\left(10-15 \mathrm{mg} \cdot \mathrm{kg}^{-1}\right.$ bw [31]) by at least a factor of 20! For healthy adult rabbits, the alveolar turnover time of PC has been reported to range at only 5-10 h [32] and, upon intratracheal injection of labelled surfactant material, the overall loss from the airspaces and the lung reached $90 \%$ and $70 \%$, respectively, after $24 \mathrm{~h}$ [33]. Although animal clearance studies have not been conducted under ARDS-like conditions, it thus seems reasonable to assume that most of the applied surfactant material was cleared from the lung within the first day after treatment. It may be speculated that some of this material may re-enter the alveolar compartment due to recycling via type-II cells.

Secondly, the $\gamma \mathrm{min}$ of the isolated large aggregate fraction, the most sensitive parameter of an alteration of the surfactant system, was significantly reduced from $\sim 23 \mathrm{mN} \cdot \mathrm{m}^{-1}$ to $\sim 13 \mathrm{mN} \cdot \mathrm{m}^{-1}$ upon treatment with Alveofact $\AA$, but control values of near 
$0 \mathrm{mN} \cdot \mathrm{m}^{-1}$ were still not achieved. The same was true for adsorption surface-tension values. This is in contrast to the far-reaching or even complete normalization of SP-B, SP-C, PC and PG concentrations in the LA fraction, and to the normalized percentage of LA. Possible underlying reasons for this incomplete restoration of the LA surface-tension lowering properties include the reduced degree of palmitoylation of the PC class contained in the LA fraction, as discussed earlier, a possibly lower degree of dipalmitoylation of PC in bovine surfactant extracts [34] as compared to healthy humans [26], and the lack of SP-A in the bovine surfactant preparation used in this study (persistently low or even decreasing percentages of SP-A were noted in the LA fraction). Moreover, a proteolytic digestion of the hydrophobic apoproteins by proteases, liberated under the inflammatory conditions in the ARDS lungs, may not be excluded, as arising fragments might similarly be captured by the antibodies and thus be detected by the ELISA procedures, as is the case with intact apoproteins. Finally, the possibility that small amounts of high molecular weight proteins, such as fibrin-oligomers which are known to severely inhibit surface activity by incorporation of surfactant components [35], might have co-pelletted during the $48,000 \times g$ centrifugation step, cannot be ruled out. The average recovery of the BALF proteins in the $48,000 \times g$ pellet was $\sim 2.7 \%$ [1]. Unfortunately, there was not enough material to perform additional experiments addressing this issue, such as investigation of surface activity after fibrinolysis, surface activity after organic solvent extraction from the LA fraction and sucrose density centrifugation.

Thirdly, the reasons for the partial loss of surfactant improvement over the subsequent $72 \mathrm{~h}$ are not clear. As discussed earlier, the rapid turnover of surfactant deposited in the alveolar compartment might be a major contributor to this finding. In addition, due to the ongoing inflammatory events in the lung tissue, the exogenous surfactant might similarly be subjected to decomposition/inhibition as the endogenous surfactant pool. Such phenomena may also underline the fact that only very minor changes in surfactant function were observed in the Survanta trial in ARDS patients, in which re-analysis of surfactant was undertaken $120 \mathrm{~h}$ post-treatment.

To conclude, this is the first study to demonstrate that bronchoscopic administration of large amounts of a natural bovine surfactant preparation in patients with severe acute respiratory distress syndrome is followed by an impressive improvement in biochemical and biophysical surfactant properties, as monitored by sequential lavage procedures. These data thus lend credit to the concept that surfactant administration is a promising therapy in acute respiratory distress syndrome, but demands large quantities of material to overcome the severe abnormalities in the endogenous surfactant system under these conditions. In addition, the development of strategies for the protection of exogenous surfactant against inhibitory mechanisms faced upon delivery to the alveolar compartment may be warranted.

\section{References}

1. Günther A, Siebert C, Schmidt R, et al. Surfactant alterations in severe pneumonia, acute respiratory distress syndrome, and cardiogenic lung edema. Am J Respir Crit Care Med 1996; 153: 176-184.

2. Gregory TJ, Longmore WJ, Moxley MA, et al. Surfactant chemical composition and biophysical activity in acute respiratory distress syndrome. J Clin Invest 1991; 88: 1976-1981.

3. Hallman M, Spragg R, Harell JH, Moser KM, Gluck L. Evidence of lung surfactant abnormality in respiratory failure. J Clin Invest 1982; 70: 673-683.

4. Pison U, Seeger W, Buchhorn R, et al. Surfactant abnormalities in patients with respiratory failure after multiple trauma. Am Rev Respir Dis 1989; 140: 10331039.

5. Schmidt R, Meier U, Yabut-Perez M, et al. Alteration of fatty acid profiles in different pulmonary surfactant phospholipids in acute respiratory distress syndrome and pneumonia. Am J Respir Crit Care Med 2001; 163: 95-100.

6. Strohmaier W, Schlag G. Experimental aspiration trauma: comparison of steroid treatment versus exogenous natural surfactant. Exp Lung Res 1993; 19: 397-405.

7. Van Daal GJ, Bos JA, Eijking EP, Gommers D, Hannappel E, Lachmann B. Surfactant replacement therapy improves pulmonary mechanics in end-stage influenza A pneumonia in mice. Am Rev Respir Dis 1992; 145: 859-863.

8. Lewis JF, Veldhuizen RA, Possmayer F, et al. Altered alveolar surfactant is an early marker of acute lung injury in septic adult sheep. Am J Respir Crit Care Med 1994; 150: 123-130.

9. Schermuly R, Schmehl T, Günther A, Grimminger F, Seeger W, Walmrath D. Ultrasonic nebulization for efficient delivery of surfactant in a model of acute lung injury. Impact on gas exchange. Am J Respir Crit Care Med 1997; 156: 445-453.

10. Berggren P, Lachmann B, Curstedt T, Grossmann G, Robertson B. Gas exchange and lung morphology after surfactant replacement in experimental adult respiratory distress syndrome induced by repeated lung lavage. Acta Anaesthesiol Scand 1986; 30: 321-328.

11. Gortner L, Bernsau U, Hellwege HH, Hieronimi G, Jorch G, Reiter HL. A multicenter randomized controlled clinical trial of bovine surfactant for prevention of respiratory distress syndrome. Lung 1990; 168: 864-869.

12. Gregory TJ, Steinberg KP, Spragg R, et al. Bovine surfactant therapy for patients with acute respiratory distress syndrome. Am J Respir Crit Care Med 1997; 155: $1309-1315$.

13. Spragg RG, Gilliard N, Richman $\mathrm{P}$, et al. Acute effects of a single dose of porcine surfactant on patients with the adult respiratory distress syndrome. Chest 1994; 105: 195-202.

14. Walmrath D, Günther A, Ghofrani HA, et al. Bronchoscopic surfactant administration in patients with severe adult respiratory distress syndrome and sepsis. Am J Respir Crit Care Med 1996; 154: 57-62.

15. Walmrath D, Grimminger F, Pappert $\mathrm{D}$, et al. Bronchoscopic administration of bovine natural surfactant in ARDS and septic shock: impact on gas exchange and haemodynamics. Eur Respir J 2002; 19: $805-810$. 
16. Bernard GR, Artigas A, Brigham KL, et al. The American-European Consensus Conference on ARDS. Definitions, mechanisms, relevant outcomes, and clinical trial coordination. Am J Respir Crit Care Med 1994; 149: 818-824.

17. Bligh EG, Dyer WJ. A rapid method of total lipid extraction and purification. Can J Biochem Physiol 1959; 37: 911-917.

18. Rouser G, Fleischer S, Yamamoto A. Two-dimensional thin layer chromatographic separation of polar lipids and determination of phospholipids by phosphorus analysis of spots. Lipids 1970; 5: 494 496.

19. Gustavsson L. Densitometric quantification of individual phospholipids. Improvement and evaluation of a method using molybdenum blue reagent for detection. J Chromatogr 1986; 375: 255-266.

20. Wright RS. A reagent for the non-destructive location of steroids and some other lipophilic materials on silica gel thin-layer chromatograms. $J$ Chromatogr 1971; 59: 220-221.

21. Krämer HJ, Schmidt R, Günther A, Becker G, Suzuki $Y$, Seeger W. ELISA technique for quantification of surfactant protein B (SP-B) in bronchoalveolar lavage fluid. Am J Respir Crit Care Med 1995; 152: 15401544.

22. Schmidt R, Steinhilber W, Ruppert C, et al. An ELISA technique for quantification of surfactant apoprotein (SP)-C in bronchoalveolar lavage fluid. Am J Respir Crit Care Med 2001; 165: 470-474.

23. Veldhuizen $\mathrm{R}$, Inchley $\mathrm{K}$, Hearn SA, Lewis JF, Possmayer F. Degradation of surfactant-associated protein B (SP-B) during in vitro conversion of large to small surfactant aggregates. Biochem J 1993; 295: 141-147.

24. Enhorning G. Pulsating bubble technique for evaluating pulmonary surfactant. J Appl Physiol 1977; 43: 198-203.

25. Seeger W, Günther A, Thede C. Differential sensitivity to fibrinogen inhibition of SP-C- vs. SP-B-based surfactants. Am J Physiol 1992; 262: L286-L291.

26. Schmidt R, Seeger W, Günther A. Alteration of molecular species of phosphatidylcholine from human pulmonary surfactant in severe pneumonia and idiophathic pulmonary fibrosis. Am J Respir Crit Care Med 1997; 155: A217.

27. Veldhuizen RAW, McCaig LA, Akino T, Lewis JF. Pulmonary surfactant subfractions in patients with the acute respiratory distress syndrome. Am J Respir Crit Care Med 1995; 152: 1867-1871.

28. Günther A, Schmidt R, Feustel A, et al. Surfactant subtype conversion is related to loss of surfactant apoprotein B and surface activity in the large surfactant aggregates-experimental and clinical studies. Am J Respir Crit Care Med 1999; 159: 244-251.

29. Seeger W, Grube C, Günther A, Schmidt R. Surfactant inhibition by plasma proteins: differential sensitivity of various surfactant preparations. Eur Respir $J$ 1993; 6: 971-977.

30. Hohlfeld JM, Struber M, Ahlf K, et al. Exogenous surfactant improves survival and surfactant function in ischaemia-reperfusion injury in minipigs. Eur Respir $J$ 1999; 13: 1037-1043.

31. Jobe A, Rider ED. Catabolism and recycling of surfactant. In: B. Robertson LM, Van Golde G, Batenburg JJ, eds. Pulmonary Surfactant. Amsterdam, Elsevier, 1992; pp. 313-338.

32. Baritussio AG, Magoon MW, Goerke J, Clements JA. Precursor-product relationship between rabbit type II cell lamellar bodies and alveolar surface-active material. Surfactant turnover time. Biochim Biophys Acta 1981; 666: 382-393.

33. Pettenazzo A, Ikegami $\mathrm{M}$, Seidner S, Jobe A. Clearance of surfactant phosphatidylcholine from adult rabbit lungs. J Appl Physiol 1988; 64: 120-127.

34. Kahn MC, Anderson GJ, Anyan WR, Hall SB. Phosphatidylcholine molecular species of calf lung surfactant. Am J Physiol 1995; 269: L567-L573.

35. Seeger W, Elssner A, Günther A, Krämer HJ, Kalinowski HO. Lung surfactant phospholipids associate with polymerizing fibrin - loss of surfactant activity. Am J Respir Cell Mol Biol 1993; 9: 213220. 